\title{
Organisation and Solubilisation of Zeolite L Crystals
}

\author{
Olivia Bossart $§$ and Gion Calzaferri^ \\ §METTLER TOLEDO Award Winner (Oral Presentation)
}

\begin{abstract}
Zeolite $L$ is a versatile host material for realising functional photonic antenna systems. Energy can be transferred within dye-loaded zeolite $L$ crystals and means have been found to transport the energy from the crystal inside to the environment or vice versa. In order to direct the energy to a target, the organisation of the zeolite crystals with respect to the environment is an important issue. Some possibilities of modifying zeolite $L$ crystals according to specific sample requirements are summarised. Zeolite $L$ material with interesting optical properties can be obtained by controlling the interaction between the crystals and their environment.
\end{abstract}

Keywords: FRET · OLED · Refractive Index Matching · Solubilisation · Zeolite

\section{Introduction}

Zeolite $\mathrm{L}$ is an aluminosilicate with onedimensional channels running along the caxis of the hexagonal crystals [1] (Fig. 1). The channels have an opening diameter of $7.1 \AA$ and reach $12.6 \AA$ at the widest place. The number of channels in a zeolite L crystal of diameter $d_{c}$ in $\mathrm{nm}$ is equal to 0.265 $\left(d_{c}\right)^{2}$. A crystal of $600 \mathrm{~nm}$ in diameter gives rise to approximately $100^{\prime} 000$ parallel channels. This vast one-dimensional channel system makes zeolite $\mathrm{L}$ a versatile host material. Fluorescent dyes of appropriate size can enter the channels. Once they are inside, they cannot pass each other or form dimers due to spatial restrictions. Hence, highly anisotropic materials with densely packed monomers can be built [2][3]. This is a prerequisite for observing efficient Förster resonance energy transfer (FRET) [4].

${ }^{\star}$ Correspondence: Prof. Dr. G. Calzaferr University of Bern

Department of Chemistry and Biochemistry

Freiestr. 3

$\mathrm{CH}-3012$ Bern

Tel.: +41316314226

Fax: +41316313994

E-Mail: gion.calzaferri@iac.unibe.ch

www.dcb.unibe.ch/groups/calzaferri/start.html

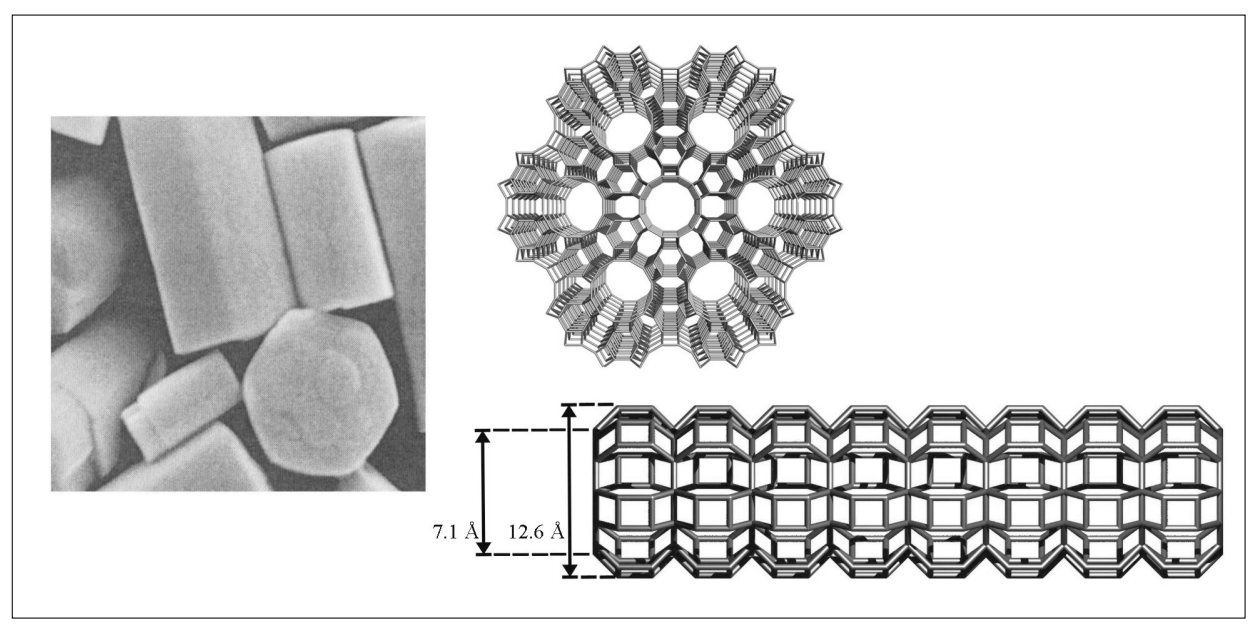

Fig. 1. Morphology and framework of zeolite L. Left: SEM image of zeolite L crystals. Top right: Framework viewed along the c-axis of a crystal with seven main channels. Bottom right: Framework side view of a main channel.

Functional photonic antenna systems have been reported, in which electronic excitation energy migrates within the dyeloaded zeolite L channels [3]. Excitation energy can either travel from the channel ends to the inside of the crystals or vice versa, depending on the sequence of the inserted donor and acceptor dyes. Monitoring the dyes inside the channels is referred to as the first stage of organisation.

The second stage of organisation is controlled by fluorescent stopcock molecules. Stopcock molecules have a tail that can enter the channels and a head that is too large to enter. They connect the inside of the crystals with their environment and enable collection of the excitation energy running through the channels on the outside of the crystals, or in- jection of energy from the outside into the crystals, respectively [5][6]. A further stage of organisation is described by the alignment of the crystals with respect to the environment and the controlled contact at the interface of the crystal and its environment. Realising this third stage of organisation is of major importance for practical applications. In this work we illustrate some zeolite L arrangements and their possible use.

\section{Third Stage of Organisation}

By inserting dye-loaded and stopcock modified crystals into an electro-luminescent polymer, light emitting diodes (LED) can be built. Upon excitation of the poly- 
mer, some excitation energy is transferred onto the stopcock molecules. From there energy is injected into the dye-loaded crystals. The resulting LED colour is composed of the polymer emission as well as the emission from the stopcocks and the incorporated dyes. Zeolite L organic light emitting diodes have the advantage of high stability and it is easy to tune the emission colour simply by changing the ratio of the participating dyes. In order to realise such diodes, a good solubilisation of the crystals in the polymer is necessary to obtain homogeneous material.

A challenging application of suitably arranged zeolites is the realisation of dyesensitised solar cells. As the only restriction in choosing the light-absorbing dyes inside the channels is the size confinement, the whole solar spectrum can be absorbed by appropriately loaded zeolites. When a monolayer of dye-loaded and stopcock modified crystals is coated onto a semiconductor, the absorbed light is transferred from the channel inside to the semiconductor via the stopcock molecules, resulting in zeolite L solar cells [7]. In order to achieve such a material, controlling the orientation of the zeolites on a surface is crucial.

Incorporating dyes into zeolite channels generally results in optically highly anisotropic single crystals. For many applications and also for spectroscopic analysis it is interesting to produce anisotropic material on a macroscopic scale. Controlling the alignment of the crystals is fundamental for attaining macroscopically polarised samples.

\section{Results and Discussion}

\section{Solubilised Zeolites}

Small zeolite crystals are hard to suspend as they have a strong tendency to stick together and form aggregates. From such suspensions, it is impossible to produce thin, homogeneous layers, for example for setting up light emitting diodes. In order to produce homogenous LEDs, suspensions containing single nanocrystals are needed. However, the interest in such suspensions goes beyond the preparation of LEDs. Preventing agglomeration of the crystals and thus minimising light scattering in the visible range is also tempting for spectroscopy as well as for using dyeloaded crystals as luminescent probes [8]. In order to achieve this, the surface of small crystals of $50 \mathrm{~nm}$ in length was modified with covalently bound methoxy(dimethyl) octylsilane (Fig. 2). The long alkyl chains improve the solubilisation in a non-polar solvent. At the same time, the modified surface helps to match the refractive index of the crystals to the surrounding solvent. In Fig. 3 unmodified and modified zeolites are suspended in toluene. Beforehand, the

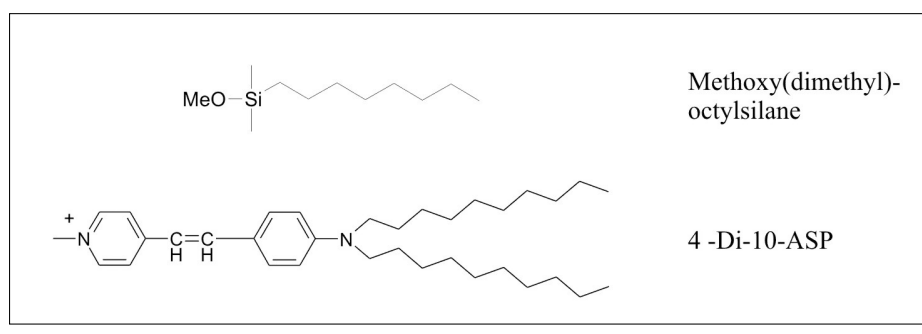

Fig. 2. Molecules used for zeolite modifications

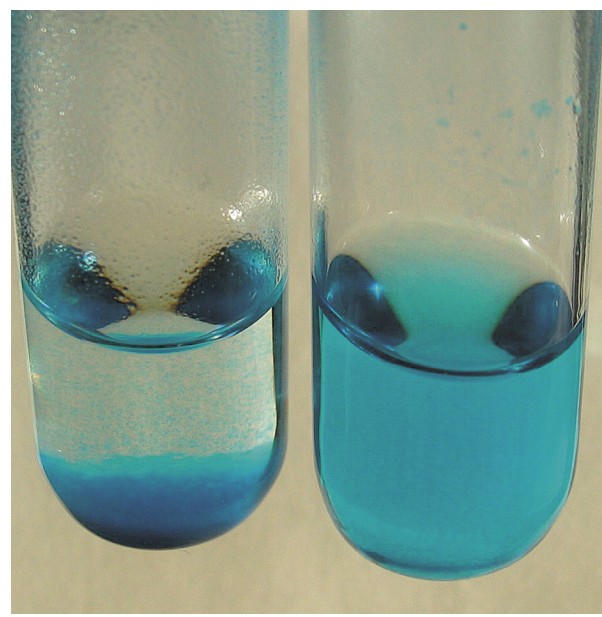

Fig. 3. Comparison of unmodified (left) and modified (right) Ox1-zeolite $\mathrm{L}$ in toluene

crystals were loaded with the blue dye oxazine $1(\mathrm{Ox} 1)$ for better visualisation. The suspension containing the modified zeolites is homogeneous and transparent, while the unmodified crystals form aggregates that sediment rapidly.

\section{Standing Zeolites}

By synthesising disc-shaped zeolites with a low aspect ratio (length to diameter) the problem of generating a monolayer of crystals standing on their base has been overcome [9]. The monolayers are prepared in such a way that the crystal bases are attached to an adequate surface, thus sealing one side of the channels. From the other side, the channels can be filled consecutively with different fluorescent dyes, resulting in a unidirectional antenna system. When the selected dyes are inserted into the channels in a suitable way, a system can be
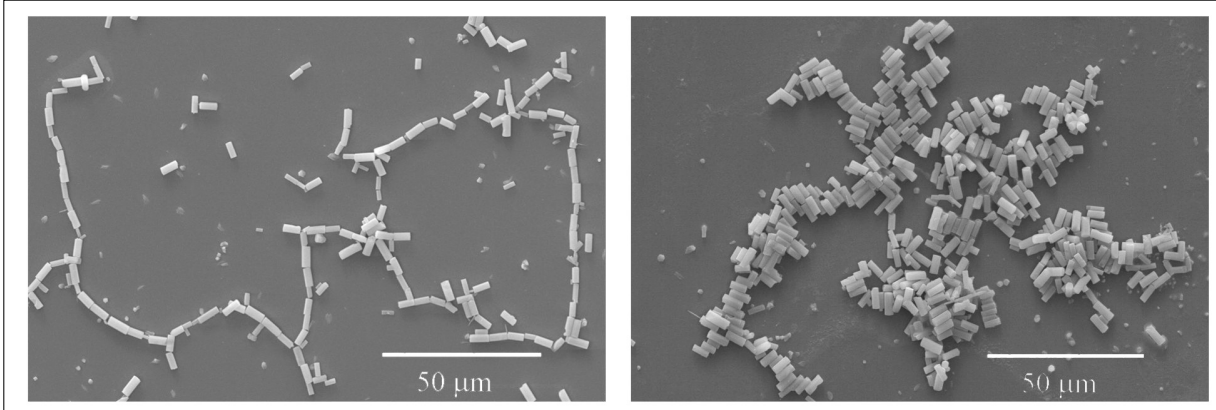

Fig. 4. SEM images of linked zeolites. Left: zeolites linked together by modifying the channel entrances. Right: Zeolites taken from the same sample after prolonged reaction time. built that absorbs the whole solar spectrum and transfers the electronic excitation energy to the sealed end. There the energy can be collected and transferred further onto a semiconductor resulting in zeolite L solar cells. So far, monolayers have been realised with zeolites exhibiting aspect ratios from 0.1 to 1 . Zeolites with a larger aspect ratio than 1 tend to lie on their side rather than stand on their base. To characterise unidirectional systems by means of fluorescence microscopy, monolayers consisting of long zeolites are favourable. 6000-nm long crystals have been synthesised, which are ideal for microscopic analysis. However, these crystals show an aspect ratio of 3 . We have observed that by modifying the zeolite surface with long alkyl chains, several zeolites stick together in a honeycomb pattern when suspended in a polar solvent. This results in large, disc-shaped clusters with a low aspect ratio which tend to stand on their base.

Approximately 6000-nm long zeolites were treated with 4-di-10-Asp (Fig. 2) in such a way that the whole crystal surface was covered with a large excess of molecules. When the sample is drop-coated onto a microscope slide directly after adding 4di-10-Asp the zeolites form vast chain structures (Fig. 4, left). A possible explanation is that due to electrostatic interactions the cationic 4-di-10-Asp is primarily attracted to the channel entrances. Hence the interaction of the alkyl chains at first only takes place there, linking together the crystals at their bases. This observation could lead to new optical material in the micrometer size range. If the reaction is not quenched at this point but the sample is stirred for further $15 \mathrm{~min}$ at room temperature and then dropcoated onto a microscope slide, chain structures are still visible, but the crystals tend to 
stick together by their coat rather than their base (Fig. 4, right). By now the crystals are probably slowly being covered with 4-di10-Asp molecules. After several hours of stirring the sample at room temperature, the whole crystal surface is covered with the modifying molecule so that several crystals are stuck together by their coats. Those building a large enough honeycomb pattern stand on their base when drop-coated onto a flat surface (Fig. 5).

\section{Aligned Zeolites}

To obtain a material with aligned crystals on a macroscopic scale, crystals loaded with yellow fluorescent pyronin (Py) were drop-coated onto a PVC plastic film. The film was then stretched to its six-fold size upon gentle heating. While heating the PVC film, the crystals melt into the polymer and are thus influenced by the mechanical force of the stretching incident. The result is that most crystals are aligned in the stretching direction (Fig. 6). Zeolites lying on top of other crystals, as well as those lying perpendicular to the stretching direction and the few standing are not affected by the stretching and stay in their original position.

\section{Conclusion}

Zeolite $\mathrm{L}$ is a versatile host material to achieve high concentrations of stable dye monomers. Such inorganic-organic hostguest materials are of considerable interest. Setting up dye-zeolite L based optical and electro-optical devices requires strict control of the crystal alignment. Once the crystals can be organised in a specific manner, many dyezeolite L based applications become feasible. We have observed that such organisation is possible by modifying the zeolite surface or by manipulating its environment. Some examples for envisaged dye-zeolite L applications are organic light emitting diodes, dye sensitised solar cells [10] and luminescent solar concentrators [11].

\section{Experimental Section}

The solubilisation experiments were carried out with $30 \mathrm{~nm}$ crystals, while all other samples were prepared with large zeolites approx. $6000 \mathrm{~nm}$ in length. The zeolites were synthesised as described in [12]. Dye loading of the crystals was carried out as described in [3].

\section{Zeolite solubilisation}

Typically $5 \mathrm{mg}$ of dye-loaded zeolite was suspended in $1 \mathrm{ml}$ of toluene (Merck, p.a.) in a Teflon tube. An excess $(20 \mu \mathrm{l})$ of methoxy(dimethyl)octylsilane (Aldrich, 98 $\%$ ) was added and the suspension heated at $70{ }^{\circ} \mathrm{C}$ overnight.

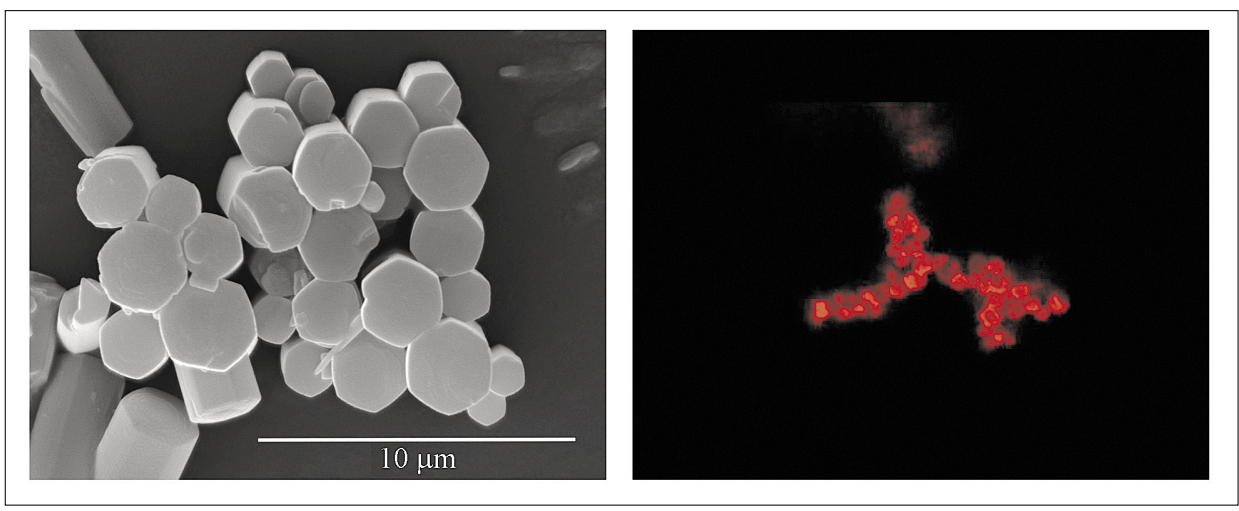

Fig. 5. Standing zeolites. Left: SEM image of standing zeolite L crystals. Right: Fluorescence microscopy image of Ox1-loaded, standing crystals.
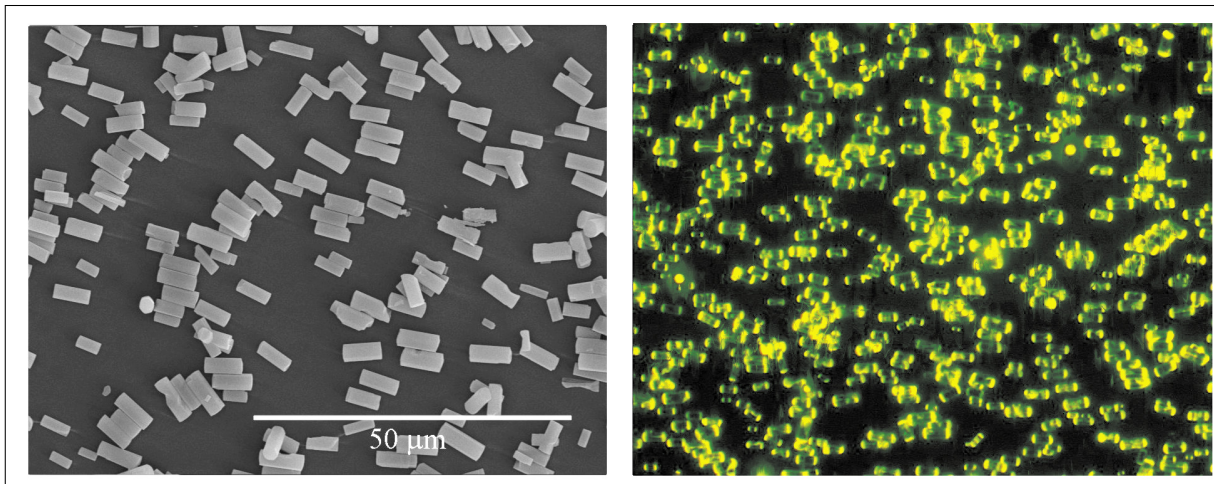

Fig. 6. Aligned zeolites. Left: SEM image of stretched PVC loaded with zeolite crystals. Right: Fluorescence microscopy image of Py-zeolite L crystals, aligned on PVC. The Py molecules are located at the channel ends due to the short insertion time of the dye.

\section{Standing zeolites}

Typically $5 \mathrm{mg}$ of dye-loaded zeolite was suspended in $2 \mathrm{ml}$ of water. An excess of 4di-10-Asp (Molecular Probes) dissolved in ethanol (absolute) was added. Instantaneously $100 \mu \mathrm{l}$ of the suspension was drop-coated onto a microscope slide to observe the linked zeolites. The suspension was stirred at $\mathrm{rt}$ for $12 \mathrm{~h}$. The standing zeolites were achieved by drop-coating $100 \mu \mathrm{l}$ of this suspension onto a glass plate $1 \mathrm{~cm}$ in diameter.

\section{Aligned zeolites}

Dye-loaded zeolites suspended in water were drop-coated onto a PVC (Fluka, high molecular weight) film. The PVC film was then stretched to its six-fold size at approximately $80{ }^{\circ} \mathrm{C}$.

\section{Acknowledgements}

This work was subsidised by the Swiss National Science Foundation NFP 47 (4047-057481).

Received: January 20, 2006

[1] a) C. Baerlocher, W.M. Meier, D.H. Olson, Atlas of zeolite Framework Types, 5th ed. Elsevier, Amsterdam, 2001; b) T. Ohsuna, B. Slater, F. Gao, J. Yu, Y. Sakamoto, G. Zhu, O. Terasaki, D.E.W. Vaughan, S. Qiu, C.R. Catlow, Chem. Eur. J. 2004, 10, 5031; c) O. Larlus, V.P. Valtchev, Chem. Mater. 2004, 16, 3381; d) C.S. Carr, D.F.
Shantz, Chem. Mater. 2005, 17, 6192.

[2] a) M. Ganschow, C. Hellriegel, E. Kneuper, M. Wark, C. Thiel, G. Schulz-Ekloff, C. Bräuchle, D. Wöhrle, Adv. Funct. Mater. 2004, 14, 269; b) G. Schulz-Ekloff, D. Wöhrle, B. van Duffel, R.A. Schoonheydt, Micropor. Mesopor. Mater. 2002, 51, 91; c) J. Caro, F. Marlow, M. Wübbenhorst, Adv. Mater. 1994, 6, 413 .

[3] G. Calzaferri, S. Huber, H. Maas, C. Minkowski, Angew. Chem. 2003, 115, 3860; Angew. Chem. Int. Ed. 2003, 42, 3732.

[4] T. Förster, Ann. Phys. 1948, 6, 55.

[5] H. Maas, G. Calzaferri, Angew. Chem. 2002, 114, 2389; Angew. Chem. Int. Ed. 2002, 41, 2284.

[6] O. Bossart, L. De Cola, S. Welter, G. Calzaferri, Chem. Eur. J. 2004, 10, 5771.

[7] S. Huber, G. Calzaferri, ChemPhysChem 2004, 5, 239.

[8] A. Devaux, Z. Popović, O. Bossart, L. De Cola, A. Kunzmann, G. Calzaferri, Micropor. Mesopor. Mater. 2006, 90, 69.

[9] A. Zabala Ruiz, H. Li, G. Calzaferri, submitted for publication.

[10] G. Calzaferri, O. Bossart, D. Brühwiler, S Huber, C. Leiggener, M.K. Van Veen, A Zabala Ruiz, C.R. Chimie 2006, 9, 214.

[11] J.S. Batchelder, A.H. Zewail, T. Cole, Applied Optics 1979, 18, 3090.

[12] A. Zabala Ruiz, D. Brühwiler, T. Ban, G. Calzaferri, Monatshefte für Chemie $\mathbf{2 0 0 5}$, 136, 77. 